This is a provisional PDF only. Copyedited and fully formatted version will be made available soon.

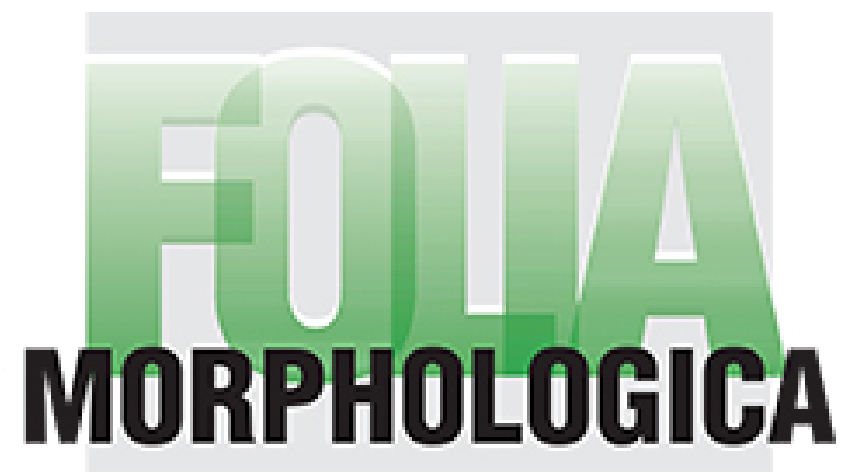

ISSN: 0015-5659

e-ISSN: $1644-3284$

\title{
A new type of the coracobrachialis muscle
}

Authors: N. Zielinska, P. Aragonés, J. R. Sañudo, P. Łabętowicz, R. S. Tubbs, Ł. Olewnik

DOI: $10.5603 / F M . a 2022.0021$

Article type: Case report

Submitted: $2021-10-31$

Accepted: $2021-12-14$

Published online: 2022-02-28

This article has been peer reviewed and published immediately upon acceptance.

It is an open access article, which means that it can be downloaded, printed, and distributed freely, provided the work is properly cited.

Articles in "Folia Morphologica" are listed in PubMed. 


\section{A new type of the coracobrachialis muscle}

N. Zielinska et al., A new type of the coracobrachialis muscle

N. Zielinska ${ }^{1}$, P. Aragonés ${ }^{2,3}$, J.R. Sañudo ${ }^{3}$, P. Łabętowicz ${ }^{1}$, R.S. Tubbs ${ }^{4-9}$, Ł. Olewnik ${ }^{1}$

${ }^{1}$ Department of Anatomical Dissection and Donation, Medical University of Lodz, Poland

${ }^{2}$ Department of Orthopedics Surgery. Hospital Santa Cristina, Madrid, Spain

${ }^{3}$ Department of Human Anatomy and Embryology, Facultad de Medicina, Universidad Complutense de Madrid, Spain

${ }^{4}$ Department of Neurosurgery, Tulane University School of Medicine, New Orleans, LA, United States

${ }^{5}$ Department of Neurosurgery and Ochsner Neuroscience Institute, Ochsner Health System, New Orleans, LA, United States

${ }^{6}$ Department of Anatomical Sciences, St. George’s University, Grenada

${ }^{7}$ Department of Neurology, Tulane University School of Medicine, New Orleans, LA, United States

${ }^{8}$ Department of Structural and Cellular Biology, Tulane University School of Medicine, New Orleans, LA, United States

${ }^{9}$ Department of Surgery, Tulane University School of Medicine, New Orleans, LA, United States

Address for correspondence: Łukasz Olewnik, PhD, Department of Anatomical Dissection and Donation, Medical University of Lodz, Poland, e-mail: lukasz.olewnik@umed.lodz.pl

\section{ABSTRACT}

The coracobrachialis muscle belongs to the anterior group of the brachial region. Its main functions are flexion and adduction at the glenohumeral joint. It is highly morphologically variable, especially in the number of bellies, place of origin or insertion, and its relationship to 
the musculocutaneous nerve. Accessory structures associated with the coracobrachialis muscle include the coracobrachialis brevis or coracobrachialis longus muscle. The present case describes a three-headed coracobrachialis muscle with two such additional structures. One of these has a tendinous origin connected to the periosteum and located on the surgical neck of the humerus. Its insertion is fused with the third head of the coracobrachialis muscle. The other has a proximal attachment fused with the capsule of the shoulder joint, and its distal attachment is fused with the third head of the coracobrachialis muscle in place of its connection with the short head of the biceps brachii. This could result in better stabilization of the glenohumeral joint; on the other hand, it could limit operational access during treatment of subscapularis tears.

Key words: coracobrachialis muscle, accessory muscle, case report, coracocapsularis longus, coracobrachialis brevis, morphological variation, embryological development, embryogenesis

\section{INTRODUCTION}

The anterior group of the brachial region consists of the coracobrachialis (CBM), biceps brachii (BBM), and brachialis (BM) muscles. The origin of the CBM is located on the coracoid process, and the short head of the BBM (SHBB) has a proximal attachment in the same place [1-3]. It is worth mentioning that in some cases these two structures create a common junction [1]. The insertion of the CBM is located on the medial surface and border of the body of the humerus, usually referred to as an anteromedial surface. Its distal attachment is between the proximal attachments of the triceps brachii and the BM. Three parts of the CBM can be distinguished: proximal (from the coracoid process), middle (larger than the first), and distal, the largest and most superficial part [4]. The CBM is supplied by the brachial artery, or more precisely by its muscular branches. Innervation is delivered by the musculocutaneous nerve (MCN), which arises from the lateral cord of the brachial plexus [13].

The main functions of this muscle are flexion and adduction at the glenohumeral joint. Its proximal attachment allows it to participate in stabilization of the humeral head (mainly anterior glenohumeral stability) [1, 2]. The subscapularis muscle (SM) has a similar function 
$[5,6]$. The CBM also prevents deviation of the arm from the frontal plane during abduction [1]. This muscle not only fulfils those anatomical functions, it can also be used in procedures such as breast reconstruction after mastectomy or treatment of facial palsy. One of the most important conditions for performing such operations is good vascularization [7, 8].

The CBM is highly morphologically variable [2, 3, 7-11]. The most interesting variation is the occurrence of additional structures, not only the presence of additional head but also, very rarely, distinct muscles. The literature includes descriptions of structures such as the coracocapsularis muscle $[4,12]$, the coracobrachialis brevis muscle $[4,13,14]$, the minor coracobrachial muscle of Cruveilhier [15], the coracobrachialis longus muscle [3, 4, 7, 11], and others [7]. They differ in their place of attachment and location in relation to the CBM [7]. Some variations of the CBM are associated with the MCN. Anatomically, this nerve should pierce the CBM, but in some cases it is located close to the muscle without piercing it $[3,8,10,11,16-18]$. A very rare morphological variation is fusion of the CBM with the medial surface of the end of the pectoralis major muscle [19].

Some CBM variations can be clinically significant because of possible compression of different nerves or blood vessels [2, 3, 8, 10,17]. Branches of the brachial plexus can potentially be compressed, leading for example to paralysis and hypoesthesia of the flexor group of the forearm [10]. However, the accessory variants of the muscle are not only pathological; as mentioned above, the CBM can be used during breast reconstruction after mastectomy. There should be no objection to using extra muscles such as the coracocapsularis for this procedure $[7,17]$.

The present report describes a three-headed variant of the CBM, which originated as a common junction with the SHBB from the coracoid process. Moreover, there were two additional structures. One of these had a tendinous origin connected with the periosteum and located on the surgical neck of the humerus. Its insertion was fused with the third head of the CBM. The other had a proximal attachment fused with the capsule of the shoulder joint, and its distal attachment was fused with the third head of the CBM in place of its connection with the short head of the biceps brachii. Knowledge of the morphological variability of this muscle is essential for clinicians. To our knowledge, this is the first description of such a case.

\section{CASE REPORT}


A 99 year old, male cadaver was subjected to routine anatomical dissection for research and teaching purposes at the University Complutense of Madrid. Dissection of the left upper limb revealed morphological variations related to the CBM.

In the present case, the CBM consisted of three heads. All three created a common junction with the SHBB, which functioned as a proximal attachment located on the coracoid process. At the point of origin the width was $11.11 \mathrm{~mm}$ and the thickness was $5.71 \mathrm{~mm}$. The distal attachment of all the heads of the CBM was located on the anteriomedial surface of the humerus; it was $6.15 \mathrm{~mm}$ wide and $1.81 \mathrm{~mm}$ thick.

As mentioned above, the first head was attached with the SHBB. The length of the first belly was $72.60 \mathrm{~mm}$. At the point of departure from the SHBB the width was $4.11 \mathrm{~mm}$ and the thickness $1.40 \mathrm{~mm}$.

The second head could be recognized as a main belly. From the point of the junction with the SHBB its length was $136.16 \mathrm{~mm}$. At the same point it was $8.99 \mathrm{~mm}$ wide and 1.89 mm thick.

The third head was really interesting. Its proximal attachment was also represented by a common junction with the SHBB. At the point of connection with the SHBB, the width was $4.64 \mathrm{~mm}$ and thickness $1.59 \mathrm{~mm}$. However, this head was connected with two anomalous structures. The length of the third head between the place of division into these two morphological variants and its distal attachment was $34.81 \mathrm{~mm}$.

The first additional structure was represented by a tendinous slip attached to the periosteum in the surgical neck of the humerus. At that location it was $1.57 \mathrm{~mm}$ wide and 0.35 mm thick. The distal attachment of this structure was fused with the third head of the CBM, and at this point of division it was $3.98 \mathrm{~mm}$ wide and $0.49 \mathrm{~mm}$ thick. The length of this variant muscle from its origin to fusion with the third head was $39.14 \mathrm{~mm}$.

The second structure was a proximal accessory band represented only by a muscular part, which was attached to the capsule of the shoulder joint. Its origin was $3.46 \mathrm{~mm}$ wide and $1.95 \mathrm{~mm}$ thick. Its distal attachment was fused with the third head of the CBM where it connected with the SHBB. The length from the origin to the fusion with the CBM was 65.41 $\mathrm{mm}$. 


\section{DISCUSSION}

The CBM is highly morphologically variable [2, 3, 7-11]. This is best demonstrated in the classification system by Szewczyk et al. [1], who identified three types with subtypes distinguished by different proximal attachments, and two types distinguished by different insertions. There were cases of CBMs originating by one single belly from the coracoid process. There were also cases of CBMs with two heads, assigned to appropriate subtypes depending on whether both heads started on the coracoid process, or one on the coracoid process and the other from the SHBB. There were also cases of a CBM with three heads, two of which originated from the coracoid process and the third from the SHBB [1].

Two types were distinguished according to the insertion of the CBM. In the first, a single belly was attached to the distal $1 / 3$ of the humerus. The second was characterized by two bellies, one attached to the distal $1 / 3$ of the humerus and the other fused with the medial head of the triceps brachii [1].

The relationship between the CBM and the MCN was also studied. In some cases the MCN pierced the muscle belly, but there were also cases in which it passed between the heads of the CBM [1].

Although the system created by Szewczyk et al. [1] is the latest classification of the CBM, variabilities were observed around one hundred and fifty years ago. For example, Wood [12] was the first to notice that the CBM can consist of three parts. The origin of the upper part was located on the coracoid process and the insertion was fused with the capsule of the shoulder joint. This structure is usually called the CBM superior, or brevis, or ratotor humeri [12]. The middle part was attached to the mid-portion of the humerus and called the CBM proprius or medius. The third and lowest part was attached to the internal condyloid ridge, the internal intermuscular septum, or the trochlea, and it was called the CBM longus. Interestingly, the middle and the lowest part are fused and together to create the CBM; the CBM brevis is rather rare $[4,12]$.

Kyou-Jouffroy et al. [20] divided the CBM into three portions. All of them had a proximal attachment on the coracoid process, but they differed in the location of their distal attachments. The first part had an insertion on the medial epicondyle of the humerus, and this structure was called the CBM longus or superficialis. The second was attached to the humeral diaphysis and was called the CBM medius. The last was inserted on to the humeral neck and was called the CBM profundus or brevis [20]. 
The CBM brevis is an interesting structure $[4,12]$. Its origin is located on the anterior surface of the coracoid process. It passes downwards and outwards and can insert on to the lesser tubercle or surgical neck of the humerus. In the present case, one of the additional structures was also attached to the surgical neck of the humerus, but we cannot call it the CBM brevis. The main difference is that the attachment of the CBM brevis on the surgical neck functions as an insertion; [4] in our case it functions as a proximal attachment. The present case also has a tendinous slip passing downwards and fused with the third head of the CBM.

The literature describes another interesting structure called the coracocapsularis muscle [4, 21]. It was recognized as a variation of the CBM brevis, the insertion of which was fused with the capsule of the shoulder joint [21]. In the present case there is an additional structure attached to the capsule, but we cannot name this a coracocapsularis muscle either. The reason is the same as above: the attachment of the coracocapsularis fused with the capsule of the shoulder joint functions as an insertion [4, 21]. In our case, it functions as an origin passing downwards and fusing with the third head of the CBM.

While searching the literature for a structure that could correspond to our muscles, we found many interesting variants related to the CBM. For example, there is the coracobrachialis minor secundus [4], which typically orginates from the coracoid process and inserts to the pectioralis major tendon [12]. There are also structures in which the insertion is fused with the tendon of the latissimus dorsi. They are named the CBM brevis s. rotator humeri, le court coracobrachialis, or the minor coracobrachial muscle of Cruveilhier $[4,15]$. They all have origins on the coracoid process $[4,15]$.

Another variation was described by Chouke et al. [22]. It had a proximal accessory band originating from the conoid ligament of the clavicle and inserting on to the medial intermuscular septum [22].

The literature also describes the CBM longus (CBL), sometimes named the CBM inferior. It is a very rare variant that can insert to the humerus, medial epicondyle, fibrous band of the medial intramuscular septum, i.e. Struther's ligament, or the tendinous part of the latissimus dorsi $[3,4,9,12,17]$. There was also a case of the CBL inserted on to the olecranon [3].

Considering all the aforementioned variants of the CBM, we can conclude that there is no special name for the additional structures we present here. We found only a case report by 
Multu Catli et al. [23] in which the CBM was represented by three heads, two of which had a typical origin on the coracoid process. Their insertion was located on the middle third of the humerus. The third head had the same point of distal attachment, but its proximal attachment was fused with the capsule of the glenohumeral joint [23]. The course of this head was therefore similar to one of the additional structures in the present case, but in the latter it was also attached to the third head at the point of its connection with the SHBB.

Coexistence of three heads of the CBM and two additional structures, one of which originated from the surgical head of the humerus and the second from the capsule of the shoulder joint, has not yet been described in the literature. To our knowledge, therefore, this is the first description of such a case.

One question is: what causes such structures to appear? Possible reasons are changes during embryo development. During embryogenesis, the upper limb bud mesenchyme of the lateral plates differentiates into intrinsic muscles. The muscle primordia then fuse and a single muscle mass of the CBM is formed [24]. Not all muscle primordia fuse, but normally disappear through cell death [25]. However, if this process fails, accessory structures such as the coracocapsularis, coracobrachialis brevis, or coracobrachialis longus muscle can result. The structures described in the present case could have arisen in the same manner.

The course of these anomalous structures can have advantages. They can both contribute to better stabilization of the shoulder joint. The first additional structure, which originated from the surgical neck of the humerus and was fused with the periosteum, could potentially prevent excessive external rotation of the humerus. The second, attached to the capsule and SHBB by the third head of the CBM, could prevent excessive anterior dislocation in the upper limb, allowing the anteroposterior and mediolateral position of the humerus to be maintained. In conclusion, these anomalous structures are involved dynamically and passively in the stability of the glenohumeral joint.

However, better stabilization could entail some limitation of precise movements. To confirm this, biomechanical studies are needed [5]. Unfortunately, there are difficulties with this, the main one being that such structures are very rare. A solution could be to examine other additional structures that could potentially function in a similar way; for example, an accessory subscapularis muscle. If a case is found in which there is insertion by a tendinous slip attached to the capsule of the shoulder joint as a distinct structure, the superior migration of the humeral head and maintenance of its anteroposterior position could be limited. 
Unfortunately, such additional structures could also have disadvantages. An operation is usually needed to treat subscapularis tendon tears [5]. During surgery, arthroscopic repair should be performed [26]. Among patients with full thickness tears less than 50\%, one anchor should be made. Among patients with full thickness tears more than 50\%, two anchors are necessary [26]. This procedure should be performed very carefully to avoid, for example, completely intersecting the rotator interval tissue and coracohumeral/superior glenohumeral ligaments [27].

In the present case, one of the additional structures was fused with the capsule of the shoulder joint, which anatomically covers the tendinous insertion of the subscapularis muscle. Occurrence of such an anomaly could be related to limitations during an operation, for example because the incision is markedly limited. To facilitate detection of different morphological variations, imaging methods such as ultrasonography or computed tomography can be used. However, there is no certainty that additional structures will be detected among all patients [26].

\section{CONCLUSIONS}

The coracobrachialis muscle is highly morphologically variable. This could be because the regression of some muscle primordia is prematurely terminated. The course of the described case could be associated with advantages such as better stabilization of the glenohumeral joint. On the other hand, such variants can significantly limit operational access during treatment of subscapularis tears.

\section{Acknowledgements}

The authors sincerely thank those who donated their bodies to science so that anatomical research could be performed. Results from such research can potentially increase mankind's overall knowledge that can then improve patient care. Therefore, these donors and their families deserve our highest gratitude [28].

\section{Ethical approval and consent to participate}


The cadaver belonged to the Department of Anatomy and Embryology of the University Complutense of Madrid.

\section{Funding}

The authors have no financial or personal relationship with any third party whose interests could be positively or negatively influenced by the article's content. This research did not receive any specific grant from funding agencies in the public, commercial, or not-forprofit sectors.

Conflict of interest: None declared

\section{REFERENCES}

1. Szewczyk B, Polguj M, Paulsen F, et al (2021) A proposal for a new classification of coracobrachialis muscle morphology. Surg Radiol Anat.

https://doi.org/10.1007/s00276-021-02700-1

2. Olewnik $€$, Zielinska N, Karauda P, et al (2020) The co-occurrence of a four-headed coracobrachialis muscle, split coracoid process and tunnel for the median and musculocutaneous nerves: the potential clinical relevance of a very rare variation. Surg Radiol Anat. https://doi.org/10.1007/s00276-020-02580-x

3. Olewnik, Paulsen F, Tubbs RS, et al (2020) Potential compression of the musculocutaneous, median and ulnar nerves by a very rare variant of the coracobrachialis longus muscle. Folia Morphol (Warsz).

https://doi.org/10.5603/fm.a2020.0085

4. Bergman R, Afifi A, Miyauchi R (2017) Illustrated Encyclopedia of Human Anatomic Variations, Anatomy Atlas.

5. Zielinska N, Tubbs RS, Podgórski M, et al (2021) The subscapularis tendon: A proposed classification system. Ann Anat 233:. https://doi.org/10.1016/j.annat.2020.151615

6. Zielinska N, Olewnik $€$, Karauda P, et al (2021) A very rare case of an accessory subscapularis muscle and its potential clinical significance. Surg Radiol Anat 43:19-25. https://doi.org/10.1007/s00276-020-02531-6

7. Georgiev GP, Tubbs RS, Landzhov B (2018) Coracobrachialis Longus Muscle: Humeroepitrochlearis. Cureus 10:. https://doi.org/10.7759/cureus.2615 
8. El-Naggar MM, Al-Saggaf S (2004) Variant of the Coracobrachialis Muscle with a Tunnel for the Median Nerve and Brachial Artery. Clin Anat 17:139-143. https://doi.org/10.1002/ca.10213

9. Georgiev GP, Landzhov B, Tubbs RS (2017) A Novel Type of Coracobrachialis Muscle Variation and a Proposed New Classification. Cureus 0-6. https://doi.org/10.7759/cureus.1466

10. Garbelotti SA, Marques SR, Rocha PR, et al (2017) An unusual case of accessory head of coracobrachialis muscle involving lateral cord of brachial plexus and its clinical significance. Folia Morphol 76:762-765. https://doi.org/10.5603/FM.a2017.0033

11. Kopuz C, Içten N, Yildirim M (2003) A rare accessory coracobrachialis muscle: a review of the literature. Surg Radiol Anat 406-410. https://doi.org/10.1007/s00276002-0079-5

12. Wood J (1867) On human muscular variations and their relation to comperative anatomy. J Anat Physiol 44-59

13. Loukas M, Aqueelah H (2005) Musculocutaneous and median nerve connections within, proximal and distal to the coracobrachialis muscle. Folia Morphol (Warsz) 64:101-108

14. Bauones S, Moraux A (2015) The accessory coracobrachialis muscle: ultrasound and MR features. Skeletal Radiol 44:1273-1278. https://doi.org/10.1007/s00256-015-21531

15. Cruveilhier J (1862) Traité d'anatomie descriptive I. Asselin, Paris, Paris

16. Olewnik $€$, Zielinska N, Karauda P, et al (2021) The co-occurrence of a four-headed coracobrachialis muscle, split coracoid process and tunnel for the median and musculocutaneous nerves: the potential clinical relevance of a very rare variation. Surg Radiol Anat 43:661-669. https://doi.org/10.1007/s00276-020-02580-x

17. El-Naggar MM (2001) A study on the morphology of the coracobrachialis muscle and its relationship with the musculocutaneous nerve. Folia Morphol (Warsz) 60:217-224

18. Tatar I, Brohi R, Sen F, et al (2004) Innervation of the coracobrachialis muscle by a branch from the lateral root of the median nerve. Folia Morphol (Warsz) 63:503-506

19. Mori M (1964) Statistics on the Musculature of the Japanese. Okajimas Folia Anat Jpn 40:195-300. https://doi.org/10.2535/ofaj1936.40.3_195

20. Kyou-Jouffroy M, Lessertissur J, Saban R, Souteyrand-Boulenger J (1971) Musculature des membres, membre pectoral, groupe branchial ventral. In: Grassé PP

21. Beattie PH (1947) Description of bilateral coracobrachialis brevis muscle, with a note on its significance. Anat Rec 97:123-126. https://doi.org/10.1002/ar.1090970110

22. Chouke K (1924) Variation of the coracobraschialis muscle. Anat Rec 157-161 
23. Catli MM, Ozsoy U, Kaya Y, et al (2012) Four-headed biceps brachii, three-headed coracobrachialis muscles associated with arterial and nervous anomalies in the upper limb. Anat Cell Biol 45:136. https://doi.org/10.5115/acb.2012.45.2.136

24. Vollala VR, Nagabhooshana S, Bhat SM, et al (2008) Multiple accessory structures in the upper limb of a single cadaver. Singapore Med J 49:e254-8

25. Guha R, Satyanarayana N, Reddy C, et al (1970) Variant insertion of coracobrachialis muscle-morphological significance, embryological basis and clinical importance. J Coll Med Sci 6:42-46. https://doi.org/10.3126/jcmsn.v6i2.3616

26. Lenart BA, Ticker JB (2017) Subscapularis tendon tears: Management and arthroscopic repair. EFORT Open Rev 2:484-495. https://doi.org/10.1302/2058-5241.2.170015

27. Park YB, Park YE, Koh KH, et al (2015) Subscapularis Tendon Repair Using Suture Bridge Technique. Arthrosc Tech 4:e133-e137. https://doi.org/10.1016/j.eats.2014.11.013

28. Iwanaga J, Singh V, Ohtsuka A, et al (2021) Acknowledging the use of human cadaveric tissues in research papers: Recommendations from anatomical journal editors. Clin Anat 34:2-4. https://doi.org/10.1002/ca.23671

Figure 1. Unrecognized type of the coracobrachialis muscle. $C P$ coracoid process of the scapula $C$ capsule of the humeral joint $B B$ biceps brachii 1 first head of the coracobrachialis muscle 2 the second head of the coracorabrachialis muscle 3 third head of the coracobrachialis muscle 


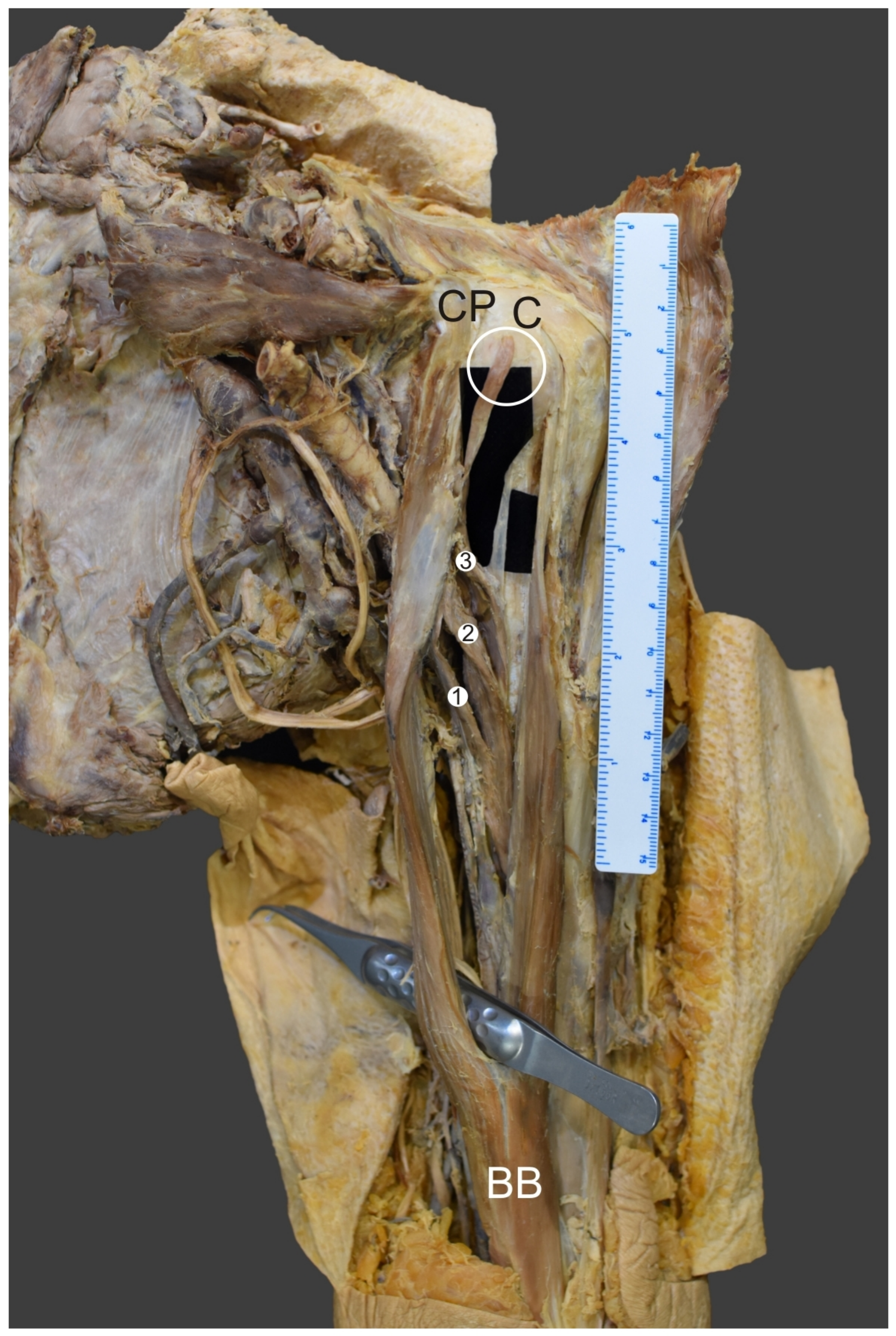

